\title{
A Study on the Strategic Reserve of China's Sports Media and Its Extension Development
}

\author{
Xiaopeng $\mathrm{Chi}^{1, \mathrm{a}}$ \\ ${ }^{1}$ Beijing Foreign Studies University, Physical Education Department, Beijing, China. \\ a Sdp618@163.com
}

Keywords: Key words: sports media, extension force, strategic reserve

\begin{abstract}
In recent years China's sports media tend to contact with advanced technology and experience of foreign countries. The Olympic Games has brought new opportunity for development of China's sports media. During the development of current sports media, we also encountered some problems, including strategic reserves of sports media, extension force and the international sports media competition, etc. Among them, strategic reserve of the sports media and its extension ability is the key point. The research object in this paper was the strategic reserve of the sports media and its extension ability. By using literature review, questionnaire and other methods, we aimed to find out the problems encountered by sports media in these areas, and put forward some reasonable suggestions.
\end{abstract}

\section{The Features of Current Sports Media in China}

The formed perfect system of sports media in China. Currently, our sports media has formed a perfect system. The audience can easily understand the instant sports information through television, network, newspapers, magazines, radio and other media. A cursory survey among college students and the surrounding community was conducted. 500 effective questionnaires were included in the final statistics. According to the statistics, most of the young men get physical information through the Internet, television, and newspapers. (Figure 1).

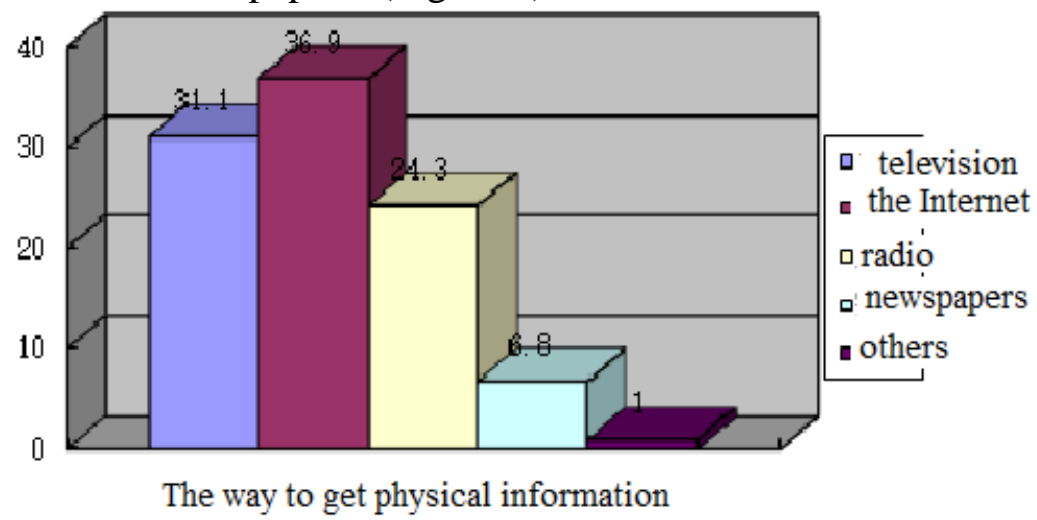

Figure 1

China's sports media is facing great challenges caused by foreign sports media. With the deepening exchanges of China's sports media and foreign advanced media, China's sports media has introduced lots of advanced foreign technology and equipment, which promotes the upgrade of domestic sports media. Therefore, the domestic sports media information has reached the international advanced level, either in the aspect of technical approach or content quality, which further enriches the domestic sports media market. While enriching the sports media market, it also brings enormous challenge to the domestic sports media survival. Compared to the domestic media, these foreign sports media often have many advantages in terms of technology, funds, international events sports broadcasting rights, which has brought the huge challenge for many domestic sports media without strong strength. 


\section{The Main Problems of Domestic Sports Media in Strategic Reserve and Extension Force}

Limited sports media strategic reserve. Lacking high-quality professional talent. Due to the increasingly refined social labor division, the professional degree of China's sports media practitioners will be higher and higher. This will require the domestic sports media should have the editing personnel with wide knowledge, but also some technical personnel related to Internet, television and other media, even some good at management and capital operation. But in our country, the sports media industry personnel structure is irrational, and the talents mastering foreign language are not enough. Those understanding management are quite limited.

Lacking adequate capital. From the current situation of the domestic sports media market, traditional media which had the advantage of news information resource is not in possession of the market. It is not the information resource owner in terms of the traditional sense, especially after the information resources become competitive products. The ultimate market winner is often the strong one with enough capital. Relying on its economic strength, they break the original pattern of competition and monopoly, purchase sports information resources of high quality, aggregate outstanding professionals, which help to control the power and voice of the final competition for sports media market. In addition, China's sports media also lack capital operation personnel and consciousness. So far, there is no separate listed sports media. This directly makes the sports media financing channels narrow, and decreases its viability and ability to resist risks falling.

Lacking strong technical strength. Compared to the sports media in western developed countries, China's sports media in technology and equipment is still relatively backward. For example, there is a huge gap between our country and the developed countries in the rebroadcast of football game. The gap of the seat and broadcast personnel's technical level caused that televising the games in China is not timely, which is difficult to increase audience's comfort while watching the game. In addition, there are still some problems in the picture library construction of domestic sports media, the construction of competition database, etc. The results of the survey are shown in the Figure 2.

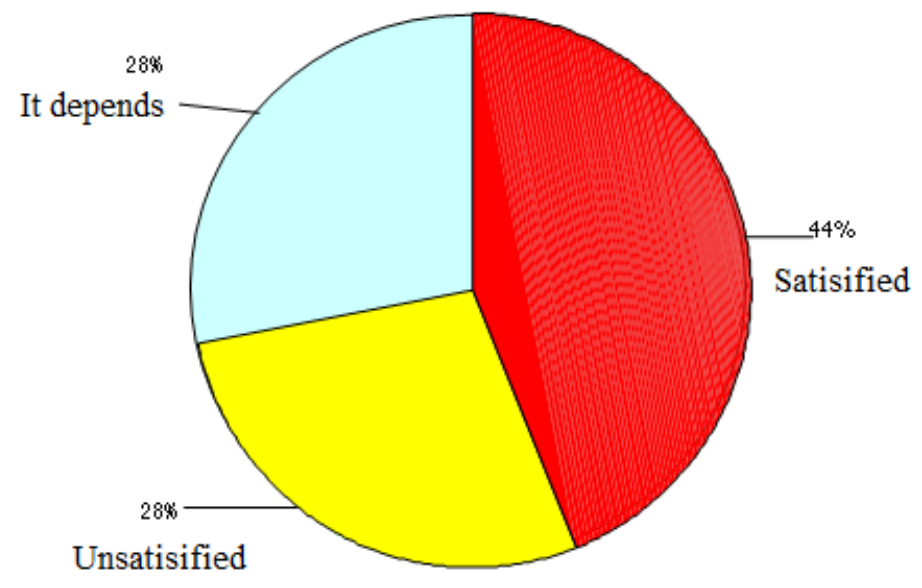

Figure 2

China's weak sports media extension ability. Single product and low utilization of information resources

The current market of China's sports media focus on the football and basketball and they pay little attention to other events. Although this is a reflection of the audience's needs, it also shows that the domestic sports media is too concentrated in the development of the related market. Current content development of sports industry can't fully meet the demand of China's sports media, which causes some potential audience. In addition, some valuable content did not attract much attention, such as sports fitness, sports leisure, sports entertainment, etc.

Weak consciousness of media cooperation. The focus attention of domestic audience is mostly foreign or international events. China's sports media must be facing the problem of information resources purchase of international sports events. The lack of high quality event and numerous domestic media cause that a part of the media will not buy the information. After purchasing the 
information, generally, the domestic media will not cooperate with other media. For other media, the lack of information leads to excessive development, which finally leads to the "double deficit situation". In addition, after the purchase of foreign or international information, the domestic sports media do not pay attention to reflect their own interests and needs to the match, which causes these events in the schedule often do not meet the demands of domestic audience.

Ignoring the three-dimensional development. The sports media in western developed countries attach great importance to the multimedia itself and the three-dimensional development. Their sports media groups generally include TV, newspapers and magazines and some other media platform. The three-dimensional development can complement each other with the mutual promotion. But the current domestic sports media ignored this aspect. The lack of talent causes that they jumped out of the main media platform, but encountered operating difficulties. This makes most of the domestic sports media lack enthusiasm to develop other media platform, and miss the benefits brought by this mode.

Paying little attention to the development of local events. The western sports media giants are mostly based on the success of one or more world influential local events. The success of British SKY owes to television broadcast of Premier League; the success of Italy "Milan sports newspaper" success owes to Italy Football League; the success of French "Equipe" owes to the tour de France and the French football league. The influence of local events often determines the national sports media influence. If a sports media put their focus on the foreign sports information, it is very difficult to become an internationally influential media. This is because the foreign media understanding of event information is difficult to surpass than local media.

Weak originality. Most of the domestic media pay much attention to the competition for foreign or international events; therefore, it is relatively difficult for domestic sports media to obtain relevant sports information. In this case, the relevant information of many domestic sports media may take direct translation of foreign media. It is in violation of the other's intellectual property, at the same time, it will also hinder their further growth.

\section{Conclusions and Suggestions for Further Development}

To strengthen the strategic reserves. (1) In the aspect of human resources, domestic sports media should actively attract professional talents in campus and society through salary, welfare and other measures. At the same time, they should try to retain the qualified employees through a variety of preferential measures, and continuously upgrade their skills through training and other various measures.

In the aspect of capital, the domestic sports media should actively expand their financing channels. Domestic sports media should adopt kinds of measures to obtain sufficient funds to increase their competitiveness, such as listing at home and abroad. Domestic sports media should actively introduce financial talents and pay higher attention to the financial capital market.

At the same time, the domestic sports media itself should also increase the investment of foreign capital while protecting its security.

In terms of technology, the domestic sports media should actively communicate with foreign advanced technology and media, learning their advanced media technology.

To strengthen the extension force. To strengthen the ability of utilizing information resources and enrich the contents of product development. For the over-exploited event information of football, basketball and other fields, the domestic sports media should avoid the repeated usage and actively develop the events surrounding information, such as tactical explanations and technical explanations, etc. For those valuable sports without much enthusiasm, such as sports fitness, sports leisure and sports entertainment, the domestic sports media should increase the development intensity, gradually guide the audience to cultivate the audience's demand and promote its benefit.

To strengthen the media cooperation. Currently, the domestic sports media should strengthen cooperation and cultivate the prosperity of the market together, so as to ensure its own long-term development prospects. In addition, during the purchase of sports information resources, the domestic sports media should also actively influence foreign event organizers, so that the related events (for 
instance, the broadcast time etc.) would be more suited to the consumption habits of domestic audience.

Adopting media pluralism and three-dimensional business model according to their own situation. Media pluralism and three-dimensional business model can make the use of its information resources. At the same time, it would obtain better benefits, and propaganda in various media between each other. This kind of management mode is suited for some large media with rich information resources. While adopting this model, the qualified domestic sports media should try to avoid the repetition of information resources. Most of the media information resources should be different. Domestic sports media should actively develop the diversity of content which is based on the features of all kinds of sports media and audience group.

To increase the supporting of local events. The success of local events is the key to the international influence of domestic sports media and the credibility among the public.

To strengthen the content originality. Domestic sports media should step up training related talents, strengthen the content originality and change the current situation of domestic sports media content which is mostly literal or guiding translation of foreign sports information. Domestic sports media should draw domestic audience's attention on the spot report of the foreign international competition and obtain the event information as much as possible to make the original foundation for the relevant content.

\section{References}

[1] Shao Peihui, Xubing. Media Strategy Management[M], Shanghai:Fudan University Press, 2003:64-65.

[2] Chen Xin. On the Development of Sports Media Industry in the USA[J].Sport Science Research, 2007 (6)

[3] Xiao Jun. Oberservation of TOM’s Intergration on Sports Media Industry. MediaObserver, 2004 (03)

[4] Guo Bin, Xu Xiaowei. Current State andDevelopmentTrends of Contemporary Sports Media. Sports Culture Guide

[5] Li Ren, Prospect of Investments in Sport Market in China[J].. Journal of Southwest University of Political Science and Law, 2005.4.

[6] Cui Dongxia, Feng Lihua. Prospect on Sports Media in China. Sports Culture Guide,2007.

[7] Huang Qiwei, Dai Jin. Prospect on Chinese Sports Media under Knowledge Economy, CCTV Sports Channel, 2007

[8] Wei Lingling, Contemplation on Legislative Deficiency of Sports Information Through Media[J]Journal of Sports Adult Education. Vol.22, No.6, 2006

[9] Zhong Humei. Mews Media Influence and Influence Economy in Relation to MediaMechanism[J]. Journal of Northeast Normal University (Philosophy and Social Sciences) 2008 (02)

[10] Wang Qing-Jun, Yang Wan-You, Self-Awareness In And Development Measures For Media Sports Communication InChina Under The Background Of Media Globalization[J].Journal Of Physical Education.Vol.15 No.1. Jan .2008

[11] Xu Li-Ping, On Survival Circumstance of Our Sports Medias at The New Times[J]. Journal Of Nanjing Institute Of Physical Education. 2007, No.6 